Kansas State University Libraries

New Prairie Press

Conference on Applied Statistics in Agriculture 2007 - 19th Annual Conference Proceedings

\title{
TRIDIMENSIONAL REGRESSION
}

Kendra Schmid

David Marx

Ashok Samal

Follow this and additional works at: https://newprairiepress.org/agstatconference

Part of the Agriculture Commons, and the Applied Statistics Commons

\section{c) $($ ) $@ \Theta$}

This work is licensed under a Creative Commons Attribution-Noncommercial-No Derivative Works 4.0 License.

\section{Recommended Citation}

Schmid, Kendra; Marx, David; and Samal, Ashok (2007). "TRIDIMENSIONAL REGRESSION," Conference on Applied Statistics in Agriculture. https://doi.org/10.4148/2475-7772.1105

This is brought to you for free and open access by the Conferences at New Prairie Press. It has been accepted for inclusion in Conference on Applied Statistics in Agriculture by an authorized administrator of New Prairie Press. For more information, please contact cads@k-state.edu. 


\title{
TRIDIMENSIONAL REGRESSION
}

\author{
Kendra Schmid , David Marx ${ }^{1}$ and Ashok Samal
}

\section{1}

Department of Statistics Department of Computer Science and Engineering

University of Nebraska-Lincoln University of Nebraska-Lincoln

\section{Lincoln, NE 68583-0963 Lincoln, NE 68588-0115}

\section{Abstract}

Shape analysis is useful for a wide variety of disciplines and has many applications. There are many different approaches to shape analysis, one of which focuses on the analysis of shapes that are represented by the coordinates of predefined landmarks on the object.

This paper introduces Tridimensional Regression, a technique that can be used for mapping images and shapes that are represented by sets of three-dimensional landmark coordinates. The degree of similarity between shapes can be quantified using the tridimensional coefficient of determination $(R)$. An experiment was conducted to evaluate the effectiveness of this technique to correctly match the image of a face with another image of the same face. These results were compared to the $R$ values obtained when only two dimensions are used, and show using three dimensions increases the ability to correctly discriminate between faces.

Key Words: bidimensional regression, nonlinear regression, face recognition, landmark data, three-dimensional shape analysis

\section{Introduction}

Tobler (1994) proposed bidimensional regression as a tool for computing the degree of similarity between two planar configurations of points and to estimate mapping relations between two objects that are represented by a set of two dimensional landmarks. Bidimensional regression is an extension of linear regression where both dependent and independent variables are represented by coordinate pairs, instead of scalar values. Specifically, Tobler (1994) suggested that bidimensional regression may be useful for comparing signatures, geographical maps, or faces. The latter was done in the context of face recognition by Shi et al. (2005).

The purpose of this work is to extend Tobler's (1994) method to Tridimensional Regression for situations when both dependent and independent variables are represented by three-dimensional coordinates. Widespread use of three-dimensional imaging devices and the use of digital elevation models (DEMs) in geographic applications make this research timely. This technique is broadly applicable to any situation where spatial configurations of three-dimensional points are compared. Specific instances where tridimensional regression may be of use are three-dimensional mapping, where altitude is measured in addition to latitude and longitude, and three-dimensional face recognition, where faces are represented by their three-dimensional coordinates. The $R$ values derived from regression allow the degree of similarity between two objects to be quantified. 2 Bidimensional Regression Nakaya (1997) defines a bidimensional regression model as:

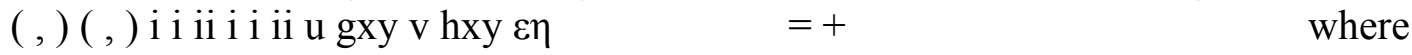
$(\mathrm{uBiB}, \mathrm{vBiB})$ is the dependent variable, $(\mathrm{xBiB}, \mathrm{yBiB})$ represent the corresponding coordinates of the independent variable, $g$ and $h$ are transformation functions used to estimate mapping relations between independent and dependent variables, and (,) i i $\varepsilon \eta$ is an error vector that is assumed to be normally and independently distributed. Both Tobler 
(1994) and Nakaya (1997) discuss obtaining estimates for parameters in $g$ and $h$ using the method of least squares so that $221^{\wedge} \wedge(()),((),) \mathrm{n}$ i i iiii i u g xyvhxy $=-+-$ $\sum$, where ${ }^{\wedge} \mathrm{g}$ and ${ }^{\wedge} \mathrm{h}$ are the transformation functions evaluated at the parameter estimates, is minimized. Here $\mathrm{n}$ is the number of landmark points used in the analysis. The normal equations are obtained in the usual manner (Tobler 1994) and by solving for $\mathrm{j} \beta$ in ${ }^{\wedge} \mathrm{T} \mathrm{T} \mathrm{j} j \mathrm{jj} X X \beta \mathrm{X} \mathrm{Y}=$, where $\mathrm{XBjB}$ is the design matrix of transformation $\mathrm{j}$ and [ ] $\mathrm{T}=\mathrm{Yu} \mathrm{v}$ is a $(2 \mathrm{n} \times 1)$ vector for the dependent variable partitioned by the coordinates, will yield the least squares parameter estimates (Draper \& Smith 1998). The design matrix $(\mathrm{XBjB})$ will depend on the transformation used and the number of parameters to estimate, hence the dimension of ${ }^{\wedge} \mathrm{j} \beta$; will also be determined by the type of transformation. 2.1 Bidimensional Regression Models Tobler (1994) proposes four bidimensional regression models, three of which are intrinsically linear and one is curvilinear. Friedman and Kohler (2003) argue that the curvilinear model may be too general for practical use and describe the linear transformations in more detail. Each of the other three transformations is linearized by reparameterization prior to solving for the parameter estimates. The three linear transformations yield the Euclidean, affine, and projective models where in each model the original coordinates are scaled, rotated, and translated. These transformations form a hierarchy with the Euclidean being the simplest (fewest parameters) and the projective the most complex (most parameters) of the models. The Euclidean model is a similarity transformation in that the overall shape remains unchanged. The coordinates are translated [ ] ( ) $12 \mathrm{~T} \alpha \alpha=\alpha$, rotated ( $\theta$ ), and isotropically scaled (s) (Dryden \& Mardia 1998), thus preserving the original shape and angles. This transformation requires the estimation of four parameters. The transformation (Tobler 1994) is $12 \operatorname{cossin} \sin \cos$ i i i i u x s v y $\alpha \theta \theta \alpha \theta \theta-$ $=+$

2221 i i i i x v y $\alpha \beta \beta \alpha \beta \beta-$ which can be reparameterized as: 11 $=+$ where the $\beta$ 's are a combination of the scale (s) and rotation $(\theta)$ parameters. The design matrix, $\mathrm{XBeB}$ (Nakaya 1997), and the parameter vector, $\beta \mathrm{BeB}$ for the Euclidean model are: $\mathrm{e} \quad 10 \mathrm{x}-\mathrm{y} \mathrm{X} 01 \mathrm{y} \times$ and [ ] $1212 \mathrm{Te} \alpha \alpha \beta \beta=\beta$. The affine model allows for $\mathrm{X}$ and $\mathrm{Y}$ coordinates to be scaled independently ( $\mathrm{sBxB}$ and sByB) and the configuration could exhibit shear $(\gamma)$ (e.g., a square may become a parallelogram; Figure 1). The affine transformation requires the estimation of six parameters. The transformation (Tobler 1994) is $120 \cos \sin 10 \sin \cos 01 \mathrm{x}$ i i y i i s u xs v y $\alpha \theta \theta \gamma \alpha \theta \theta$

$-$ $=+$

which can be reparameterized as: 121342 i i i i u x v y $\beta \beta \alpha \beta \beta \alpha$ $=+$ where the $\beta$-matrix is a combination of rotation, shear, and scale parameters. The design matrix, XBaB (Nakaya 1997), and the parameter vector, $\beta \mathrm{BaB}$ for the affine model are: $\mathrm{a}=10 \mathrm{x} \mathrm{y} 00 \mathrm{X0100 \textrm {x }}$ $\mathrm{y}$ and [ ] $121234 \mathrm{Ta} \alpha \alpha \beta \beta \beta \beta=\beta$. The projective transformation, which is the most complex, allows the size, shape, and orientation to change as a function of viewpoint (Friedman \& Kohler 2003). The projective transformation requires the estimation of nine parameters. An example of a projective transformation is shown in Figure 2. The transformation (Tobler 1994) is 111213313233 i i i i i x y u x y $\beta \beta \beta \beta \beta \beta++=++, 21$ 2223313233 i i i i i x y v x y $\beta \beta \beta \beta \beta \beta++=++$, which are converted to homogeneous coordinates 111213212223313233 " 1 i i i i u x v y t $\beta \beta \beta \beta \beta \beta \beta \beta \beta$ 
$==$. (1) The design matrix, $\mathrm{XBpB}$ and the parameter vector, $\beta \mathrm{BpB}$ for the projective model are: $\mathrm{p} \quad \mathrm{x}$ y $1000000 \mathrm{X} 000 \mathrm{x}$ y $1000000000 \mathrm{x}$ y 1 and [ ] $111213212223313233 \mathrm{Tp} \beta \beta \beta \beta \beta \beta \beta \beta \beta=\beta$, where $1(, \ldots,) \mathrm{T} \mathrm{nx} \mathrm{x}=\mathrm{x}, 1$ ( ,..,.) $\mathrm{T}$ $\mathrm{n} y \mathrm{y}=\mathrm{y}, 1 \mathrm{x}(0, \ldots, 0) \mathrm{T} \mathrm{n}=0$, and $1 \mathrm{x}(1, \ldots, 1) \mathrm{T} \mathrm{n}=1$. The fact that these are linear mappings implies that straight lines in the original space remain straight lines after the transformation. For the Euclidean and affine transformations, lines that are parallel in the original space will be mapped to parallel lines in the transformed space (Friedman \& Kohler 2003). In the Euclidean and affine transformations, the models are linearized by reparameterization, and then the normal equations can be derived in the usual manner. Once the parameters have been estimated, Friedman \& Kohler (2003) provide equations for calculating the scale and rotation values for the Euclidean transformation and the scale, shear, and rotation values for the affine transformation. The similarity of the two objects is assessed using the bidimensional correlation coefficient, $2222^{\wedge} \wedge\{()()\} 1$ $\{()()\}$ i i ii i D i i i u u vv R u u vv $-+-=--+-\sum \sum$. When using the Euclidean transformation, the value of RB2DB is the same regardless of whether [ ]T $=\mathrm{Y} \mathrm{u} \mathrm{v}$ is the dependent or independent variable (Friedman \& Kohler 2003). This is not the case when the affine or projective transformation is applied. The transformation parameter estimates differ depending on the order in which the transformations are applied, but the value of the correlation coefficient does not depend on the order of the transformations (Friedman $\&$ Kohler 2003). The equations for the projective transformation can be rewritten using homogeneous coordinates and put in matrix notation as shown in Equation(1). Homogeneous coordinates can be used with any of the models to provide a uniform framework for all transformations. For rotation, scaling and shear, the transformed coordinates can be expressed as the product of a transformation matrix and the original coordinates. For translation, however, the coordinates are derived by addition of the translation vector to the original coordinates. Use of homogeneous coordinates makes all the transformations multiplicative. This is accomplished by adding an additional coordinate $(\mathrm{t})$, called the homogeneous coordinate. The homogeneous coordinate is added purely for mathematical simplification and has no effect on the transformation of coordinates. For example, it is convenient to represent a sequence of transformations as the product of the corresponding transformation matrices. Thus, in the Euclidean and affine models, the translation parameters become multiplicative and one matrix could be used for all of the transformation parameters (Foley et al. 1995). With the projective model, the conversion is used to linearize the model, and once the object is mapped using homogeneous coordinates, the original coordinates are restored by dividing by the homogeneous coordinate, $t$. However, when this is done, the restriction placed on $t$ results in parameter estimates of $31^{\wedge} 0 \beta=, 32^{\wedge} 0 \beta=, 33^{\wedge} 1 \beta=$. Consequently, the projective transformation is reduced to the affine transformation and the results are identical. The conversion to homogeneous coordinates is adequate for determining the location of transformed points, but not for obtaining transformation parameter estimates. If left in terms of the original equations, the parameters of the projective transformation can be estimated using nonlinear regression. 3 Tridimensional Regression The bidimensional regression models proposed by Tobler (1994) can be extended to instances where threedimensional data are used for comparison. A specific instance may include spatial data where dependent and independent variables are represented by latitude, longitude, and altitude, but tridimensional regression can be useful for determining the degree of 
similarity between any two objects that are represented by three-dimensional coordinates. 3.1 Tridimensional Regression Models In this paper, the linear transformations discussed by Tobler (1994) will be extended to three dimensions. Extensions to the Euclidean, affine, and projective transformations are described in detail below where the dependent and independent variables are represented by their three-dimensional coordinates, iii uvw and iii xyz , respectively. 3.1.1

Euclidean Transformation The three-dimensional Euclidean transformation is similar to the two-dimensional case in that coordinates are simply translated, rotated, and isotropically scaled. The overall shape and the angles of the original object are preserved, and parallel lines in the original object are mapped to parallel lines in the transformed space. There is an additional translation parameter, and the rotation matrix differs depending on which axis(es) are used for the rotation. In general, the number of rotation parameters is $\mathrm{k}(\mathrm{k}-1) / 2$ where $\mathrm{k}$ is the number of dimensions. Therefore, there are three rotation parameters for the general three-dimensional Euclidean transformation. However, for instances when it is known that all three rotations are not necessary, the transformation can be reduced to one or two rotations. These special cases are discussed first. UThree-dimensional Euclidean transformation with one angle of rotation The format of the rotation matrix depends on the axis of rotation. The formats for each of the three rotations are shown below, where $\gamma$ is the angle of rotation about the $\mathrm{x}$-axis, $\theta$ is the angle of rotation about the $\mathrm{y}$-axis, and $\varphi$ is the angle of rotation about the z-axis: 1000 $\operatorname{cossin} 0 \sin \cos \gamma \gamma \gamma \gamma \quad-\quad \mathrm{XR}=, \cos 0 \sin 010 \sin 0 \cos \theta \theta \theta \theta$ - $\quad \mathrm{YR}=$, and $\cos \sin 0 \sin \cos 0001 \varphi \varphi \varphi \varphi^{-}$ $\mathrm{ZR}=$. The general form of the three-dimensional Euclidean transformation is 123 i i i i i u x v s y w z $\alpha \alpha \alpha$ $=+$

$\mathrm{R}$ where $\mathrm{R}$ is one of the rotation matrices. As in the two dimensional case, the transformation can be linearized by reparameterization, where the new transformation matrix ( $\mathrm{R}$ ) is a combination of the scale and rotation parameters. The reparameterized transformations and their normal equations follow. For rotation about the $\mathrm{x}$-axis, 12211 $0000 \beta \beta \beta \quad \quad$ ' $\quad X \mathrm{R}=, 1212321$ - i i i i i i i u x v yzw y $\mathrm{z} \alpha \alpha \beta \beta \alpha \beta \beta=+=+=++$, and deriving the normal equations in the usual manner yields: $123221222 \mathrm{~N} 0000$ () $0 \mathrm{~N} 000 \mathrm{~N} 0$ ()0 () 00 () () ) i i i i i i i i i i ii i i ii i i ii i i ii u x y z v z y w y z yz v y wz z y yz w y vz $\alpha \alpha \alpha \beta \beta$

$$
=\quad++\quad-+-
$$

$\sum \sum \sum \sum \sum \sum \sum \sum \sum \sum \sum \sum \sum \sum \sum$. For rotation about the y-axis, 1221001 $00 \beta \beta \beta \beta \quad$ Y R =, 1122321 i i i i i i i uxzvyw xz $\beta$ $\beta \alpha \alpha \beta \beta=++=+=-+$, and deriving the normal equations in the usual manner yields: $123221222 \mathrm{~N} 000 \mathrm{~N} 000$ () $00 \mathrm{~N} 0$ ()0 () 00 () () i i i i i i i i i i ii i i ii i ii i ii i x z u v y z x w x z Xz u x wz z X xz u z wx $\alpha \alpha \alpha \beta \beta$

$$
-=\quad++
$$$$
-+-
$$

$\sum \sum \sum \sum \sum \sum \sum \sum \sum \sum \sum \sum \sum \sum \sum$. For rotation about the z-axis, 12210000 $1 \beta \beta \beta \beta-\quad Z \mathrm{R}=, 1122213$ i i i i i i i uxy v x y w z $\beta$ $\beta \alpha \beta \beta \alpha=+-=++=+$, and deriving the normal equations in the usual manner yields: $123221222 \mathrm{~N} 000 \mathrm{~N} 000 \mathrm{~N} 00() 0() 0() 00()()$ i i i i i i i i i i iiiiii i i iiiii x y u y x v w z x y x yuxvy y x xyvxuy $\alpha \alpha \alpha \beta \beta$

$$
\begin{array}{cccc}
= & ++ & -+ & \sum \sum \sum
\end{array}
$$

$\sum \sum \sum \sum \sum \sum \sum \sum \sum \sum \sum \sum$. UThree-dimensional Euclidean transformation with multiple 
angles of rotation When more than one rotation is used, the reparameterization to linearize the model is not obvious; therefore, the rotation matrices remain in terms of the rotation parameters and nonlinear regression is used. The advantage of using nonlinear regression is that the rotation and scale parameters are directly estimated instead solved in terms of $\beta \mathrm{BiB}$; the disadvantage in using nonlinear regression is convergence may not be reached and starting values must be specified. The similarity of the two objects is assessed using the Pseudo-RP 2P as defined by Schabenberger \& Pierce (2002). The Pseudo- RP 2P is calculated in the same manner as RP 2P, but in general, is not guaranteed to be greater than zero. Again, the rotation matrix differs depending upon the axes of rotation. An example of a two rotation Euclidean transformation is shown below. For rotation about $\mathrm{x}$ and $\mathrm{y}$-axes, $123 \cos 0 \sin \sin \sin \operatorname{cossin} \cos \cos \sin \sin \cos \cos$ i i i i i i u x v s y w z $\alpha \theta \theta \alpha \gamma \theta \gamma \gamma \theta \alpha \gamma \theta \gamma \gamma \theta$ $=+-$

. In the general form of the threedimensional Euclidean transformation, the order in which the transformations are applied will result in different parameter estimates. Permuting this order will result in different estimates of the rotation parameters, but the measure of similarity will remain the same regardless of the order of transformations. The following system of equations shows the rotations in the order of $\mathrm{x}$-axis, $\mathrm{y}$-axis, and then z-axis: 123 cos coscossinsin sinsincoscossinsinsinsincoscossincos cossincossinsincossinsinsincoscoscos i i i i i i u x v s y w z $\alpha \theta \varphi \theta \varphi \theta \alpha \gamma \theta \varphi \gamma \varphi \gamma \theta \varphi \gamma \varphi \gamma \theta \alpha \gamma \theta \varphi \gamma \varphi \gamma \theta \varphi \gamma \varphi \gamma \theta-$

$=++-+-\quad-++3.1 .2$ Affine

Transformation The extension of the affine transformation from two dimensions into three dimensions includes additional parameters for translation, scaling, rotation, and shear. Figure 3 shows an example of a three-dimensional affine transformation. The transformed coordinates in affine transformations are given by: 111121322122233 313233 i i ii i i ii i i ii u x yz v x yz w x yz $\alpha \beta \beta \beta \alpha \beta \beta \beta \alpha \beta \beta \beta=+++=+++=+++$ and 111121322122233313233 i i i i i i x v y w z $\alpha \beta \beta \beta \alpha \beta \beta \beta \alpha \beta \beta \beta$

$$
=+
$$

Deriving the normal equations in the usual manner yields: ( ) $3322332 \mathrm{~N}$ i i i i i iiii i iiiii i iiiii x y z x x xy x z y xy y yz z xzyzz $\otimes \otimes$

$\otimes \otimes$

I $123111213212223313233 \alpha \alpha \alpha \beta \beta \beta \beta \beta \beta \beta \beta \beta$

i i uvw u x u y z v x y v z w x w y w z

\section{$\sum \sum \sum \sum \sum \sum \sum \sum \sum \sum \sum \sum \sum \sum \sum \mathrm{I} \mathbf{I} \mathbf{I}$}

$\sum \sum \sum \sum \sum \sum \sum \sum \sum \sum \sum \sum$, where

IB3B is a $3 \times 3$ identity matrix and $\otimes$ is the direct product of the two matrices, which can be solved to obtain the estimates for the transformation parameters. 3.1.3 Projective Transformation The extension of the projective transformation from two to three dimensions involves the conversion to homogeneous coordinates (Equation(2)). Additional parameters are added corresponding to the coordinate of the third dimension. In a projective transformation, the size, shape, and orientation can all change as a function of viewpoint. While this is a non-linear transformation, by using homogeneous coordinates, the model can be linearized in order to obtain the normal equations and estimate the parameters. The equations to obtain the transformed coordinates are: 11 1213144142434421222324414243443132333441424344 i i i i i i i i i i i i i i i i 
i i x y z u x y z x y z v x y z x y z w x y z $\beta \beta \beta \beta \beta \beta \beta \beta \beta \beta \beta \beta \beta \beta \beta \beta \beta \beta \beta \beta \beta \beta \beta \beta+$ $++=++++++=++++++=+++$ and 11121314212223243132333441424344 1 i i i i i i utx vty wt zt $\beta \beta \beta \beta \beta \beta \beta \beta \beta \beta \beta \beta \beta \beta \beta \beta$ $=$ Let "' i i i i i i u ut v vtw wt $===$ then, 11121314212223243132333441424344 "' 1 i i i i i i u x v y w z t $\beta \beta \beta \beta$ $\beta \beta \beta \beta \beta \beta \beta \beta \beta \beta \beta \beta$

, (2) and deriving the normal equations in the usual manner yields: $111213142122223224423132333441424344 \mathrm{~N}$ ux uy uz u vx vy vz x xyxzx $\mathrm{v}$ xy yyzy wx xz yzzz wy x y z $\beta \beta \beta \beta \beta \beta \beta \beta \beta \beta \beta \beta \beta \beta \beta \beta$

$$
\otimes=
$$

\section{$\sum \sum \sum \sum \sum \sum \sum \sum \sum \sum \sum \sum \sum \sum \sum \sum \sum \sum \sum \sum \sum \mathrm{I}$ wz wtx ty tz $\mathrm{t}$}

\section{$\sum \Sigma \Sigma \Sigma$}

$\sum \sum \sum \sum \sum \sum$, where IB4B is a $4 \times 4$ identity matrix and $\otimes$ is the direct product of the two matrices. As with the two-dimensional projective transformation, this linearization results in parameter estimates that reduce the transformation to affine. The linearization is adequate to determine the transformed points, but not for the optimization to determine the transformation parameters. Therefore, again the transformation is left in terms of the original equations and nonlinear regression is used to obtain parameter estimates. Nonlinear regression is an extension of linear regression where the expected responses are nonlinear functions of the parameters (Bates \& Watts 1988). Finding least squares estimates for linear models is straightforward as they have a closed-form solution. For nonlinear models, the least squares estimates must be found using an iterative procedure. In this paper, the Gauss- Newton algorithm is used. This iterative procedure utilizes a Taylor series expansion to find the least squares estimates (Bates \& Watts 1988). The similarity of the two objects can be assessed using the tridimensional correlation coefficient. The tridimensional correlation coefficient, RB3DB is given by: 2 $223222^{\wedge} \wedge\{()()()\} 1\{()()()\}$ i i iiii i D i i i i u u vvww R u u vvww -+ -+-=--+ $-+-\sum \sum$, which is an extension to the bidimensional correlation coefficient given by Tobler (1994). 4 Illustration An experiment was conducted to evaluate the effectiveness of tridimensional regression and its improvement over bidimensional regression. Three dimensional landmark data obtained from human faces were used for this purpose. The landmarks were obtained by placing reflective markers on the faces of subjects and tracking the coordinates as the subjects moved through a series of poses using automated software. The landmarks were adapted from Farkas (1994). They are shown in Figure 4 and described in Table 1. The landmarks were obtained for three subjects at two different sittings and with five poses per sitting. The objective was to compare RP 2P values within a subject to the RP $2 \mathrm{P}$ values between subjects using both tridimensional regression and bidimensional regression. One would expect the degree of similarity to be higher, thus a higher RP 2P value, for two samples from the same person than for samples from two different people. All pair wise RP 2P values were calculated for bidimensional and tridimensional regressions. Poses within a sitting were not compared since the markers were not removed between poses and using these poses would result in inflated RP 2P values. Thus there are 75 comparisons within a subject (five poses for each of two sittings with three subjects) for the Euclidean model and 150 for the affine and projective models (switching independent and dependent variables yields different 
results) and 300 comparisons for between subjects (Ten poses from first subject (two sittings) with ten poses from the second subject (two sittings), three pair wise comparisons) using the Euclidean model (600 for affine and projective). For each transformation, both in two and three dimensions, the distributions of RP 2P values for within and between subjects were obtained by fitting a theoretical distribution over the histograms of observed values. Overlaying these theoretical distributions allowed for the estimation of a threshold value $(\tau)$ for determining if two samples were from the same subject. RP 2 Pvalues greater than $\tau$ lead to the decision that the two samples are from the same subject while RP 2P values less than $\tau$ indicate the samples are from two different subjects. The threshold value was determined to be where the two distributions cross, as to simultaneously minimize the false positive and false negative error rates. A false positive is when samples from two different subjects are incorrectly determined to be from the same subject (an RP 2P value greater than $\tau$ for different subjects); a false negative occurs when two samples from the same subject are incorrectly determined to be from different subjects (an RP 2P value less than $\tau$ for the same subject). In addition to calculating the observed error rates, the expected error rates were found by evaluating the cumulative distribution functions of the RP 2P values at $\tau$. Table 2 summarizes the observed and expected error rates, Figures 5-7 show the within subject (dotted line) and between subject (solid line) distributions for each transformation. Table 2 shows that both the observed and expected error rates for tridimensional regression are much smaller than those for bidimensional regression using any of the three transformations. Bidimensional regression resulted in both error rates being very high; false positives often over fifty percent. Tridimensional regression shows a substantial decrease in both false positive and false negative error rates which indicates that the three-dimensional method is better at correctly matching a subject to him or herself. In this application, the Euclidean and affine transformations were comparable to one another with the affine performing slightly better. The projective transformation had the largest observed false positive rate. This result is not surprising as the flexibility of the projective transformation allows it to map objects into many other shapes. This flexibility results in the ability to match even two very dissimilar objects quite well with certain transformation parameters.

Consequently, the RP 2P values are very high for all matches. This shifts the between person distribution closer to the within person distribution which results in a larger false positive error rate. 5 Summary and Future Work Bidimensional regression, developed by Tobler (1994), is a useful tool for comparing two geometric configurations that are each represented by a set of coordinate pairs. The scale, rotation, and translation relating the two configurations can be estimated by first estimating the parameters of the transformation model. As an application of the technique, Shi et al. (2005) used bidimensional regression analysis for relating faces in landmark based face recognition. In this paper, the bidimensional technique has been extended to three dimensions. Such an extension may prove useful in the analysis of three-dimensional landmark data. The underlying foundations for tridimensional regression have been developed with different transformations: Euclidean, affine and projective. Its use is demonstrated through an application to compare human faces using three-dimensional landmarks. Results show that tridimensional regression improves the ability to correctly match objects that are represented by landmark data. Both the Euclidean and affine transformations work well to reduce the error rates. The projective transformation also shows improved error rates, 
but its flexibility may make it too general for some practical applications. This work can be extended in several different directions. The focus here was in developing the theory of tridimensional regression and conducting an initial investigation for shape matching with a feasibility experiment. An investigation with a larger amount of three- dimensional landmark data is needed to more fully understand its effectiveness. In addition to a larger scale study, it is also of interest to develop weighted bidimensional and tridimensional regression techniques which would allow some landmarks to be weighted more or less heavily than others. Weighting could greatly improve the matching ability of bidimensional and tridimensional regression.

Acknowledgements

The authors wish to thank Dr. Jordan Green of the University of Nebraska-Lincoln for his help in obtaining the three-dimensional landmark data used for this research.

References

Bates, D. M. \& Watts, D. G. 1988 Nonlinear Regression Analysis and its Applications. New York: John Wiley \& Sons.

Casella, G. \& Berger, R. 2002 Statistical Inference $\left(2^{\text {nd }}\right.$ ed.). Pacific Grove, CA: Duxbury.

Draper, N. R. \& Smith, H. 1998 Applied Regression Analysis (3 ed.). New York: John Wiley \& Sons.

Dryden, I. L., \& Mardia, K. B. 1998 Statistical Shape Analysis. New York: John Wiley \& Sons.

Farkas, L. G. 1994 Anthropometry of the Head and Face (2 ${ }^{\text {nd }}$ ed.). New York: Raven Press.

Foley, J. D., van Dam, A., Feiner, S. K., \& Hughes, J. F. 1995 Computer Graphics: Principles and Practice in C (2 ed.). Boston: Addison-Wesley.

Friedman, A. \& Kohler, Bernd. 2003 Bidimensional regression: assessing the configural similarity and accuracy of cognitive maps and other two-dimensional data sets. Psychological Methods, 8 (4), 468-491.

Kramer \& Richter. 2005 Projective Transformation. Available on the World Wide Web at http://doc.cinderella.de/tiki-index.php?page=Projective+Transformation . Last Accessed on June 5, 2007.

Nakaya, T. 1997 Statistical inferences in bidimensional regression models. Geographical Analysis, 29, 169-186.

Schabenberger, O., \& Pierce, F. J. 2002 Contemporary statistical models for the plant and soil sciences. New York: Taylor \& Francis Group.

Shene, Ching-Kuang. Geometric transformations. Available on the World Wide Web at http://www.cs.mtu.edu/ shene/COURSES/cs3621/NOTES/geometry/geo-tran.html . Last Accessed on June 5, 2007.

Shi, J., Samal, A., \& Marx, D. 2005 Face recognition using landmark-based bidimensional regression. IEEE Comput. Soc. Proc. On Data Mining.

Tobler, W. 1994 Bidimensional regression. Geographical Analysis, 26, 187-212.

Figure 1: Example of a Bidimensional Affine Transformation Figure 2: Example of a Bidimensional Projective Transformation (ABCD $\rightarrow$ FGHE) (Kramer \& Richter 2005) Figure 3: Example of a Tridimensional Affine Transformation (Shene 2007) Figure 4: Landmarks used for evaluating Tridimensional Regression tr sci sci $n$ ex en prn ls ch ch ex en li go go gn gn li ch ls prn n sci tr en ex go ac jm sa pa sba 
Figure 5: Within and Between person $\mathrm{R}^{2}$ for bidimensional (1) and tridimensional (r) Euclidean transformation

Figure 6: Within and Between person $\mathrm{R}^{2}$ for bidimensional (l) and tridimensional (r) affine transformation

Figure 7: Within and Between person $\mathrm{R}^{2}$ for bidimensional (1) and tridimensional (r) projective transformation

Table 1: Description of landmarks used for evaluation (adapted from Farkas (1994))

\begin{tabular}{|l|l|}
\hline tr & The point on the hairline in the midline of the forehead. \\
\hline go & The most lateral point on the mandibural angle close to the bony gonion. \\
\hline gn & The lowest median landmark on the lower border of the mandible. \\
\hline en & The point at the inner commissure of the eye fissure. \\
\hline ex & The point at the outer commissure of the eye fissure. \\
\hline sci & The highest point on the upper boarder in the midportion of each eyebrow. \\
\hline n & The midpoint of both the nasal root and the nasalfrontal structure. \\
\hline prn & The most protrudent point of the apex nasi. \\
\hline ac & The most lateral point in the curved base line of each ala. \\
\hline ls & The midpoint of the upper vermillion line. \\
\hline li & The midpoint of the lower vermillion line. \\
\hline ch & The point located at each labial commissure. \\
\hline sa & The highest point of the free margin of the auricle. \\
\hline sba & The lowest point of the free margin of the ear lobe. \\
\hline pa & The most posterior point on the free margin of the ear. \\
\hline jm & The most protrudent point of the muscle when the jaw is clenched. \\
\hline
\end{tabular}

Table 2: Error rates for each transformation

\begin{tabular}{|c|l|r|r|r|r|}
\hline \multicolumn{3}{|c}{ Bidimensional Regression } & \multicolumn{3}{c|}{ Tridimensional Regression } \\
\hline \multicolumn{3}{|c|}{ False Positive } & \multicolumn{1}{c|}{ False Negative } & False Positive & False Negative \\
\hline \multirow{3}{*}{ OEuclidean } & bserved & $59.7 \%$ & $36.0 \%$ & $19.3 \%$ & $12.0 \%$ \\
\cline { 2 - 6 } & Expected & $51.9 \%$ & $33.6 \%$ & $16.7 \%$ & $9.0 \%$ \\
\hline \multirow{3}{*}{ Affine } & Observed & $57.5 \%$ & $38.7 \%$ & $17.0 \%$ & $3.3 \%$ \\
\cline { 2 - 6 } & Expected & $49.0 \%$ & $37.2 \%$ & $14.9 \%$ & $7.5 \%$ \\
\hline \multirow{3}{*}{ OProjective } & bserved & $56.8 \%$ & $35.3 \%$ & $23.5 \%$ & $7.3 \%$ \\
\cline { 2 - 6 } & Expected & $51.2 \%$ & $34.5 \%$ & $18.4 \%$ & $16.2 \%$ \\
\hline
\end{tabular}

